ARTICLE

\title{
Color-tunable ultralong organic room temperature phosphorescence from a multicomponent copolymer
}

Long $\mathrm{Gu}^{1}$, Hongwei Wu${ }^{1}$, Huili Ma², Wenpeng $\mathrm{Ye}^{2}$, Wenyong $\mathrm{Jia}^{2}$, He Wang${ }^{2}$, Hongzhong Chen ${ }^{1}$, Nan Zhang ${ }^{1}$, Dongdong Wang (1) ${ }^{1}$, Cheng Qian ${ }^{1}$, Zhongfu An (1) ${ }^{2 凶}$, Wei Huang ${ }^{2,3 凶}$ \& Yanli Zhao (i) ${ }^{1 凶}$

Functional materials displaying tunable emission and long-lived luminescence have recently emerged as a powerful tool for applications in information encryption, organic electronics and bioelectronics. Herein, we present a design strategy to achieve color-tunable ultralong organic room temperature phosphorescence (UOP) in polymers through radical multicomponent cross-linked copolymerization. Our experiments reveal that by changing the excitation wavelength from 254 to $370 \mathrm{~nm}$, these polymers display multicolor luminescence spanning from blue to yellow with a long-lived lifetime of $1.2 \mathrm{~s}$ and a maximum phosphorescence quantum yield of $37.5 \%$ under ambient conditions. Moreover, we explore the application of these polymers in multilevel information encryption based on the color-tunable UOP property. This strategy paves the way for the development of multicolor bio-labels and smart luminescent materials with long-lived emission at room temperature.

\footnotetext{
${ }^{1}$ Division of Chemistry and Biological Chemistry, School of Physical and Mathematical Sciences, Nanyang Technological University, 21 Nanyang Link, Singapore 637371, Singapore. ${ }^{2}$ Key Laboratory of Flexible Electronics \& Institute of Advanced Materials, Jiangsu National Synergistic Innovation Center for Advanced Materials, Nanjing Tech University, 30 South Puzhu Road, Nanjing 211816, P. R. China. ${ }^{3}$ Shaanxi Institute of Flexible Electronics, Northwestern Polytechnical University, 127 West Youyi Road, Xi'an 710072, P. R. China. ${ }^{凶}$ email: iamzfan@njtech.edu.cn; iamwhuang@njtech.edu.cn; zhaoyanli@ntu.edu.sg
} 
R oom-temperature phosphorescence with ultralong emission lifetime has garnered tremendous interests in recent years due to their unique long-lived and rich excited-state features $^{1-4}$, which can be used in a wide array of potential technological applications, spanning from decorative and emergency displays to information anti-counterfeiting and bioapplications ${ }^{5-10}$. Nevertheless, this fascinating luminescence phenomenon was considered an exclusive property of inorganic materials in the past decades on account of the inefficient intersystem crossing (ISC) and intensive non-radiative decay of organic luminophores under ambient conditions. The intrinsic disadvantages of these inorganic counterparts such as the harsh preparation conditions, the scarcity of metal resource and high toxicity for bio-application have led to the search for metal-free alternatives $^{11}$. In the recent years, alternative molecule design rules and enhanced strategies such as crystal engineering ${ }^{12-16}$, host-guest doping ${ }^{17}$, metal-organic frameworks (MOFs) ${ }^{18}$, $\mathrm{H}$-aggregation ${ }^{19,20}$, and others ${ }^{21-23}$ are proposed to achieve ultralong organic room-temperature phosphorescence (UOP) through improving the ISC and suppressing the non-radiation decay of triplet excitons. Generally, constructing a rigid environment through crystal engineering is the most common approach to obtain the long-lived phosphorescence emission at room temperature $^{24}$. However, the crystal-based UOP materials have problems of reproducibility, processability, and flexibility, which greatly hinder the development of crystal-based UOP materials for practical applications ${ }^{25,26}$. To overcome these fundamental hurdles, special attention has been paid to the development of organic polymeric materials capable of emitting ultralong phosphorescence at room temperature. Although significant breakthroughs have been achieved by prolonging the lifetime of UOP based on polymeric materials through the construction of a rigid polymer microenvironment via homopolymerization ${ }^{27}$ and radical binary copolymerization ${ }^{28}$, as well as embedding small molecules into a rigid polymer matrix ${ }^{29}$, research on color tunability of polymer-based UOP in a single polymer under ambient conditions has not been reported.
Color-tunable luminescent materials have gained considerable attention owing to their potential applications in multicolor display, polychromatic imaging agents in biological applications, information encryption, and anti-counterfeiting ${ }^{30-33}$. Various strategies have been developed to create materials with colortunable emission in both small molecule and polymeric systems, which include modulating the composition of materials, changing the molecular conformations, and regulating the molecular packing mode in crystals ${ }^{34-39}$. Despite the successful developments of fluorescence materials with multicolor emission by rational molecular design and engineering, it is still a great challenge to obtain room-temperature phosphorescence materials that demonstrate color tunability in response to an external stimulus, such as light, electric fields, humidity, and pressure.

Inspired by the common methods of achieving multicolor emission in organic light-emitting diodes through the incorporation of luminophores with different emission colors into one polymer as well as the construction of multiple emitting centers in a single-component molecular crystal to obtain multicolor room-temperature phosphorescence emission under different excitation wavelengths, we propose that excitation wavelengthresponsive UOP may be obtained by conjugating multiple UOP emitting centers onto a polymer backbone through radical crosslinked copolymerization under ambient conditions (Fig. 1a, b).

\section{Results}

Photophysical properties of PDNA polymer. To validate our hypothesis, we designed and prepared a multicomponent copolymer by radical cross-linked copolymerization of acrylic acid and multiple luminophores (Fig. 1c). Polyacrylic acid (PAA) contains numerous carbonyl and hydroxyl groups, which not only enhance spin-orbit coupling by improving the ISC from singlet-to-triplet excited states for generating the triplet excitons but also restrict molecular motion by constructing an intermolecular/intramolecular hydrogen bonding network between the polymer matrix and luminophores, suppressing the non-radiative decay of excited triplet state. In order to achieve the color-tunable UOP emission a

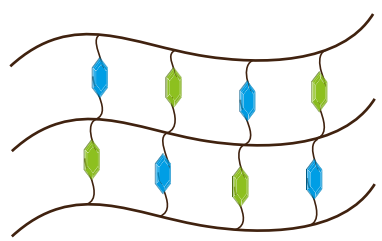

Multicomponent copolymer b

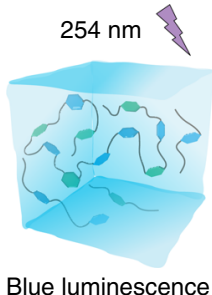

Blue luminescence

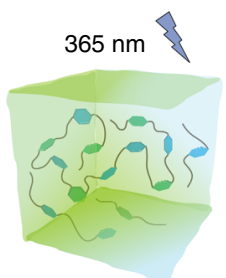

Yellow luminescence

C
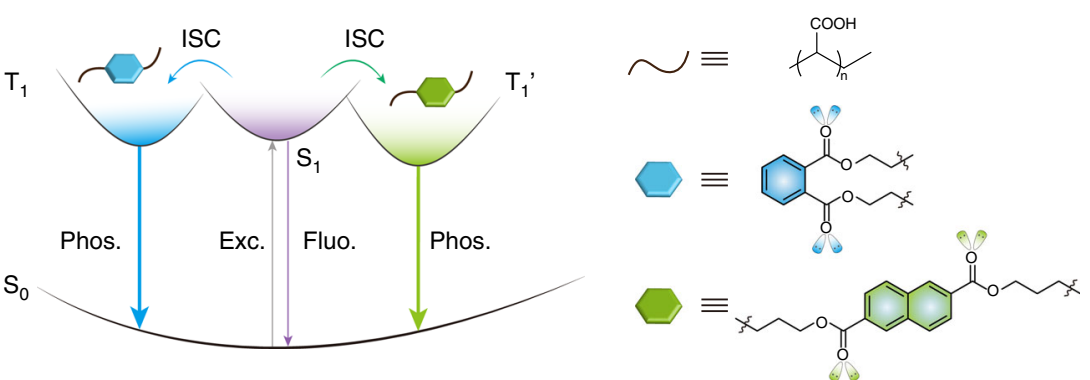

Fig. 1 Schematic illustration of the color-tunable UOP multicomponent copolymer. a Network structure of multicomponent copolymer by radical crosslinking copolymerization. The brown line and blue and yellow hexagons represent PAA chains and various luminophores, respectively. $\mathbf{b}$ Excitationdependent UOP of multi-component copolymer under ambient conditions. The polymer exhibits color-tunable UOP emission upon changes in the excitation wavelength. c Proposed mechanism of color-tunable UOP at room temperature. Multiple triplet excited state $T_{1}$ and $T_{1}$ generating from different excited singlet states $S_{1}$ stem from the various luminophores through ISC. The rigid polymer microenvironment effectively restricts molecular motion for ultralong phosphorescence emission under ambient conditions. 
a

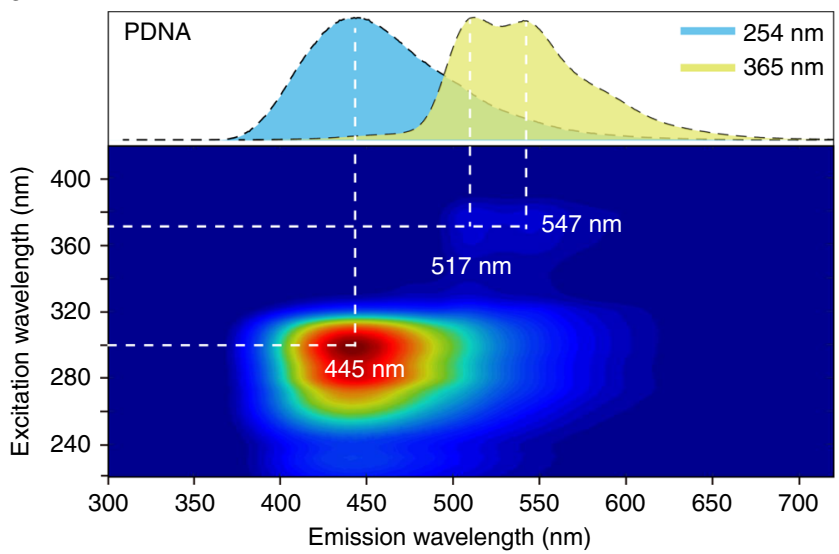

b

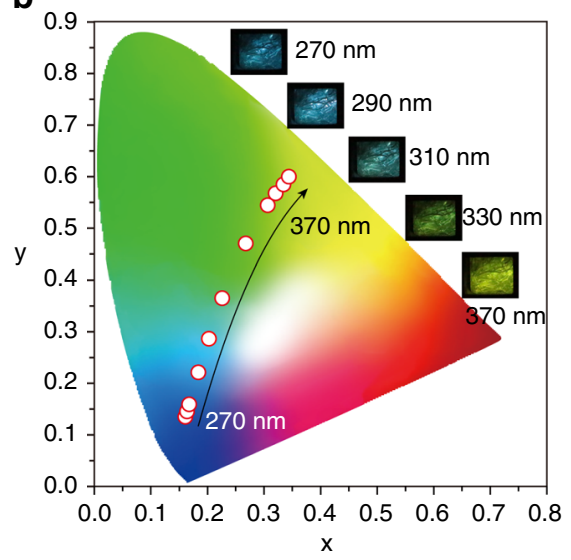

C
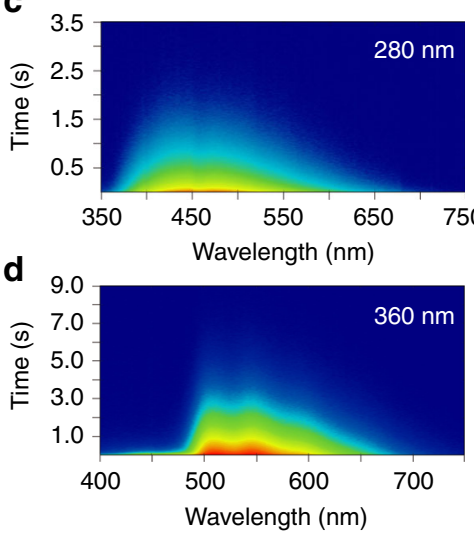

e

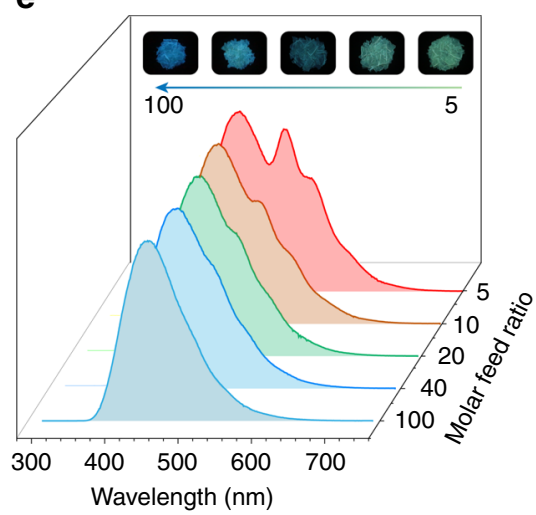

f

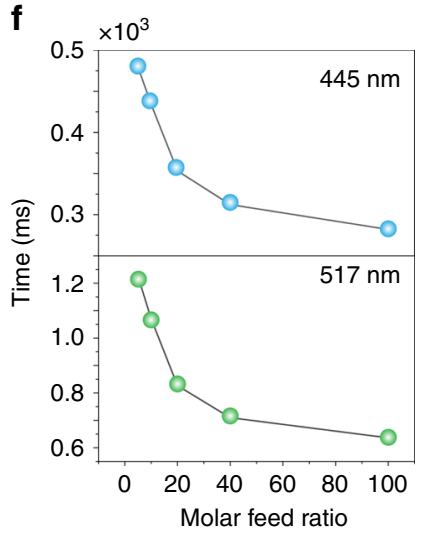

Fig. 2 Photophysical properties of multicomponent copolymer at room temperature. a Excitation-phosphorescence mapping of polymer film PDNA under ambient conditions. The inset displays the phosphorescence spectra of the transparent film recorded at room temperature excited by $254 \mathrm{~nm}$ (blue) and $365 \mathrm{~nm}$ (yellow). b CIE chromaticity diagram for polymer PDNA with excitation varied from 270 to $370 \mathrm{~nm}$. Inset: long-lived luminescence photographs of polymer film PDNA excited at 270, 290, 310, 330 and $370 \mathrm{~nm}$, respectively. c, d Time-resolved emission spectra of PDNA at room temperature upon excitation at 280 and $360 \mathrm{~nm}$, respectively. e Normalized phosphorescence spectra and the ultralong phosphorescence photographs (inserted images) of multicomponent copolymers with different molar feed ratio of MND:MDP:AA =1:5:1000 (PDNA-5), 1:10:1000 (PDNA-10), 1:20:1000 (PDNA-20), 1:40:1000 (PDNA-40), and 1:100:1000 (PDNA-100) excited at $254 \mathrm{~nm}$. f Phosphorescence lifetime of PDNA with different molar feed ratios of PDNA-5, PDNA-10, PDNA-20, PDNA-40, and PDNA-100 at 445 (top) and $517 \mathrm{~nm}$ (bottom) when excited at 254 and $360 \mathrm{~nm}$ at room temperature, respectively.

under different excitation wavelengths, chromophores with different degrees of conjugation were incorporated into the PAA chains though functionalized alkyl chains, which may avoid undesirable complications caused by energy transfer or aggregation. Meanwhile, the formation of a cross-linked polymer network may further enhance the rigidity of polymer matrix for generating long-lived phosphorescence emission under ambient conditions.

As a proof of concept, we synthesized a copolymer (PDNA) by radical cross-linked copolymerization of acrylic acid (AA), vinylfunctionalized naphthalene (MND), and benzene (MDP) using 2-azoisobutyronitrile (AIBN) as an initiator (Supplementary Methods and Supplementary Figs. 1-3). The chemical structures were characterized by nuclear magnetic resonance (NMR) spectroscopy (Supplementary Figs. 4-10). The molar feed ratio of MND/MDP/AA was $1 / 200 / 10,000$. The polymer PDNA shows number-average molecular weights $(\mathrm{Mn})$ of $67.3 \mathrm{~K} \mathrm{Da}$ (Supplementary Table 1). As anticipated, blue long-lived luminescence of approximately $4 \mathrm{~s}$ was observed by the naked eye from the transparent polymer film PDNA after switching off the ultraviolet (UV) lamp of $254 \mathrm{~nm}$ under ambient conditions (Supplementary Fig. 11 and Supplementary Movie 1). Notably, when the excitation wavelength was changed from 254 to $365 \mathrm{~nm}$, a distinctly different yellow color long-lived emission that lasted for approximately $9 \mathrm{~s}$ could be observed after switching off the UV lamp, exhibiting an excitation wavelength-responsive ultralong luminescence characteristic.

To further investigate on this interesting color-tunable longlived luminescence phenomenon, we performed the excitationphosphorescence spectra measurements of the PDNA polymer film at room temperature. As illustrated in Fig. 2a, when the excitation wavelength varied from 220 to $420 \mathrm{~nm}$, the main emission center of polymer PDNA showed a significant bathochromic shift from 445 to $547 \mathrm{~nm}$, accompanied by the long-lived luminescence color turned from blue to yellow under ambient conditions (Supplementary Fig. 12). Furthermore, Commission International de l'Eclairage (CIE) coordinate diagram of the PDNA film was calculated from their phosphorescence spectra excited at corresponding wavelengths to demonstrate the color change of PDNA (Fig. 2b). As the excitation wavelength increased from 270 to $370 \mathrm{~nm}$, the variation in long-lived luminescence color spanned from blue to yellow, demonstrating good linearity on the CIE coordinate. In addition, similar color-tunable long-lived luminescence phenomenon for polymer PDNA can also be observed at 
low temperature $(77 \mathrm{~K})$ and oxygen atmosphere (Supplementary Figs. 13 and 14), indicating that the external environment has little influence on the performance of color-tunable long-lived emission. Meanwhile, the steady-state photoluminescence spectra of polymer PDNA also exhibit an excitation wavelength-responsive luminescence property under ambient conditions (Supplementary Fig. 15).

Analysis of the time-resolved phosphorescence emission spectra of polymer PDNA at room temperature provided insights on its long-lived luminescence property. As shown in Fig. 2c, d, the main emission bands at 445, 517, and $547 \mathrm{~nm}$ in PDNA show an ultralong lifetime of 428,949 , and $1096 \mathrm{~ms}$ with a phosphorescence quantum yield of $23.2 \%$ upon excitation at 280 and $360 \mathrm{~nm}$, respectively (Supplementary Fig. 16 and Supplementary Table 2), indicating phosphorescence emission characteristics. This phenomenon was further confirmed through oxygen-quenching phosphorescence experiments. Compared with the phosphorescence spectra of PDNA in nitrogen atmosphere, it was found that the intensity of ultralong emission peaks displayed a significant reduction under an oxygenated environment, which is the property of phosphorescence emission (Supplementary Fig. 17).

Effect of different molar feed ratio on UOP. The ratio variation of different luminophores may have a great influence on the luminescence color in the polymer. Hence, we systematically explored the effects of the variation in molar feed ratio of MND/ $\mathrm{MDP} / \mathrm{AA}$ on the photophysical properties in the resulting copolymer. A series of multicomponent copolymers with different molar feed ratio ranging from 1:5:1000 to 1:100:1000 were prepared. As the molar feed ratio of MND/MDP/AA changed from 1:5:1000 (PDNA-5), 1:10:1000 (PDNA-10), 1:20:1000 (PDNA-20), 1:40:1000 (PDNA-40) to 1:100:1000 (PDNA-100), the phosphorescence intensity of bands at 517 and $547 \mathrm{~nm}$ decreased gradually under excitation at $254 \mathrm{~nm}$, resulting in the hyperchromatic shift of long-lived luminescence color from green to blue under ambient conditions (Fig. 2e and Supplementary Fig. 18), displaying molar feed ratio dependent UOP property. Nevertheless, when the excitation wavelength was at $365 \mathrm{~nm}$, these polymers all displayed long-lived yellow luminescence after switching off the excitation source (Supplementary Fig. 19). Moreover, when the molar ratio of MDP increased from 5 to 100 , the lifetime of these polymers at 445 and $517 \mathrm{~nm}$ showed a significant decrease from 479.7 and $1222 \mathrm{~ms}$ to 282 and $636.2 \mathrm{~ms}$, respectively (Fig. 2f and Supplementary Figs. 20-22 and Supplementary Table 3 ). This decease of lifetime in polymers might be assigned to the intensive non-radiative transition and quenching of triplet excitons by motion and collision of phosphors in polymer matrix, resulting from the incorporation of abundant luminophores into polymer chains. In addition, the polymer PDNA also displays time-dependent phosphorescence emission, with the delay time prolonged from 5 to $500 \mathrm{~ms}$ and the phosphorescence emission band at $445 \mathrm{~nm}$ decreased gradually, leading to the color change on the CIE chromaticity coordinates (Supplementary Fig. 23)

Proposed mechanism for color-tunable UOP. To probe the mechanism underlying this color-tunable phosphorescence emission in multicomponent copolymer, the phosphorescence excitation spectra of polymer film PDNA at 445 and $517 \mathrm{~nm}$ were recorded at room temperature. As shown in Fig. 3a, significantly different excitation spectra at 445 and $517 \mathrm{~nm}$ were observed in polymer PDNA. When the excitation wavelength is in the range from 230 to $302 \mathrm{~nm}$, blue long-lived luminescence is more intense than the yellow emission. In contrast, phosphorescence emission was dominated by the yellow luminescence when the excitation wavelength ranges from 303 to $390 \mathrm{~nm}$. The ratiometric variation of phosphorescence emission intensity at 445, 517, and $547 \mathrm{~nm}$ upon changes in the excitation wavelength leads to color-tunable long-lived luminescence, which agrees with the excitationdependent ultralong organic phosphorescence spectra. More importantly, from the analysis of the excitation spectra, it was revealed that the blue and yellow emission bands at 445 and $517 \mathrm{~nm}$ stem from two different excited triplet states. Considering that the two luminophores were introduced into the PAA chains, we thus speculated that the two triplet state emission may have originated from the monomer phosphorescence luminescence of vinyl-functionalized luminophores $\mathrm{MDP}$ and $\mathrm{MND}$ in this copolymer.

To verify this, the photophysical properties of monomer MDP and MND were investigated in both solid state and diluted solution (Supplementary Note 1 and Supplementary Fig. 24). In the solid state, the samples MDP and MND displayed intensive blue fluorescence emission at 367 and $382 \mathrm{~nm}$ as well as at 387 and $405 \mathrm{~nm}$, respectively. However, no phosphorescence signal could be detected at room temperature (Supplementary Fig. 25). In addition, the phosphorescence properties of the monomers MDP and MND in dilute 2-methyltetrahydrofuran (m-THF) solution at $77 \mathrm{~K}$ were studied (Fig. 3b, top). It was found that the emission shape and major peaks of monomer MDA and MNA at 424,505 , and $537 \mathrm{~nm}$ under excitation at 280 and $360 \mathrm{~nm}$ in $\mathrm{m}$ THF solution were similar to the blue and yellow phosphorescence emission at 445,517, and $547 \mathrm{~nm}$ in the multicomponent copolymer PDNA under ambient conditions, respectively. This result indicated that the blue and yellow ultralong phosphorescence emission in PDNA was attributed to the single-molecule phosphorescence of derivatives of benzene and naphthalene. In order to accurately investigate the photophysical property of the monomers MDP and MND in polymer, we synthesized another two kinds of two-component copolymers PDA and PNA containing single luminophore, by radical cross-linked copolymerization of monomers MDP, MND, and AA with the molar feed ratio 1:1000, respectively, and their photophysical properties were studied by phosphorescence spectra and lifetime profiles at room temperature (Supplementary Note 2 and Supplementary Figs. 26-29). As expected, the phosphorescence bands at around 443, 510, and $543 \mathrm{~nm}$ excited at corresponding excitation wavelengths of 280 and $360 \mathrm{~nm}$ in the polymers PDA and PNA agreed with the blue and yellow emission in polymer PDNA at room temperature (Fig. 3b, bottom and Supplementary Figs. 30 and 31). These control polymers all exhibited a long-lived luminescence with the lifetimes of 463,842 , and $835 \mathrm{~ms}$ at the corresponding emission bands (Supplementary Table 4).

As control experiments, to eliminate the possibility that the color-tunable UOP emission in polymer was a result of various aggregation emissions of polymer chains upon changes in the excitation wavelength, we also performed the measurement of excitation-phosphorescence spectra on binary copolymers PDA and PNA (Fig. 3c). It was shown that, when the excitation wavelength was varied from 200 to $400 \mathrm{~nm}$, the ultralong organic phosphorescence was dominated by the blue and yellow emission in polymers PDA and PNA ascribing to the isolate phosphorescence emission of MDP and MND, respectively. No excitationdependent UOP behavior was observed. Therefore, the variation in the excitation wavelength does not result in various aggregated state phosphorescence emission in polymer chains but rather modulates the intensity ratio of emission peaks at 445,517 , and $547 \mathrm{~nm}$, leading to the color-tunable UOP in polymer PDNA. This illustration was further confirmed by the wide-angle X-ray scattering of the polymer PDNA. As shown in Fig. 3d, except the two broad scattering bands at around $1.27 \AA\left(16.76^{\circ}\right)$ and $2.41 \AA\left(35.60^{\circ}\right)$ were observed, which can be attributed to the scattering bands of PAA (Supplementary Fig. 32$)^{40}$, no other $\pi-\pi$ 

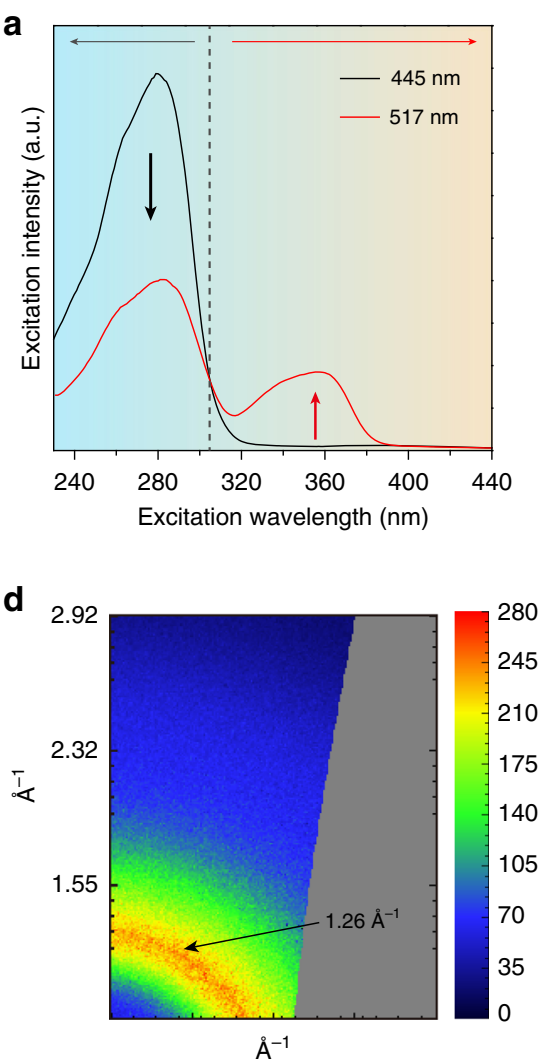
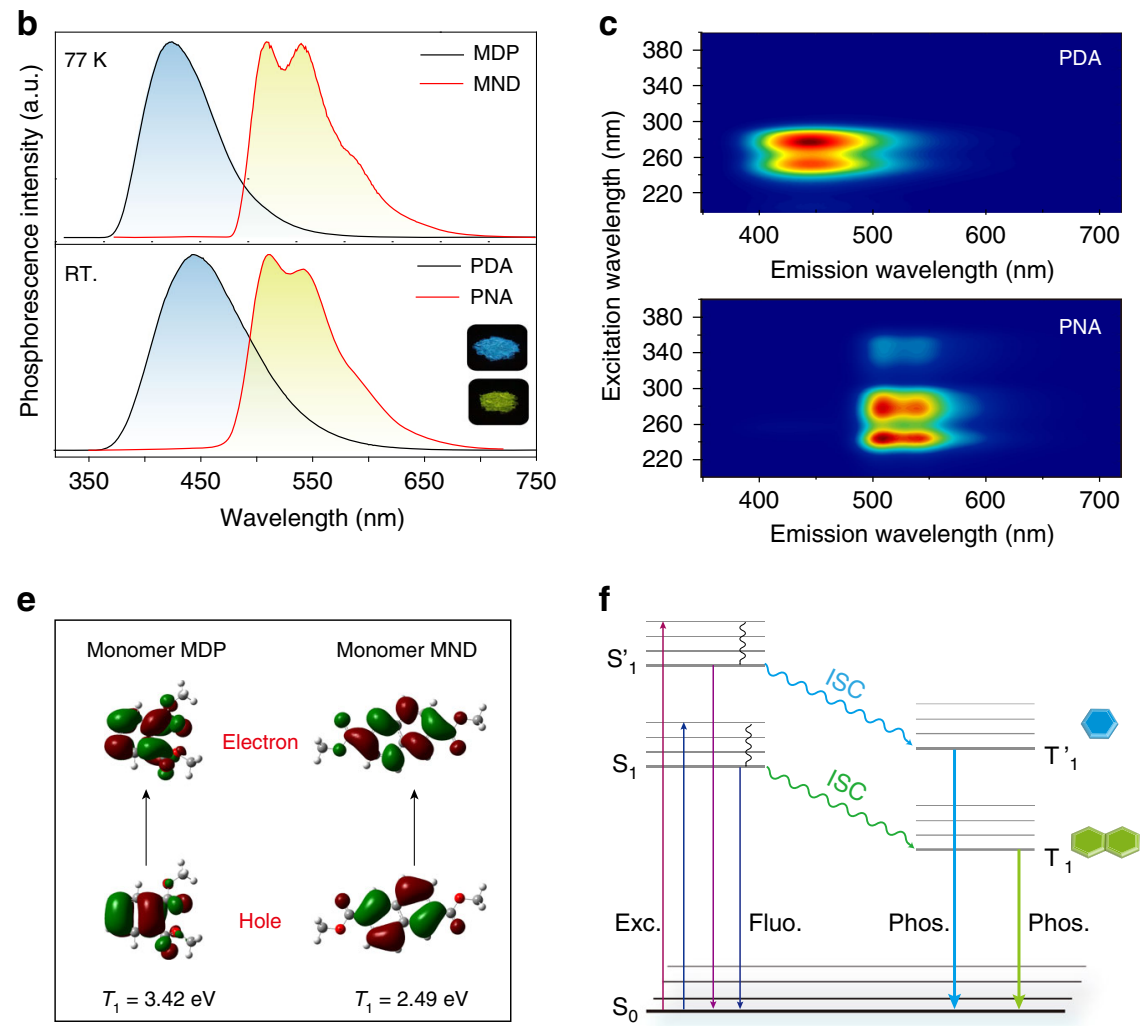

Fig. 3 Mechanism of color-tunable UOP in multicomponent copolymer at room temperature. a Phosphorescence excitation spectra in polymer PDNA at 445 and $517 \mathrm{~nm}$. b Normalized phosphorescence spectra of monomer MDP and MND in 2-methyltetrahydrofuran (1× $10^{-5} \mathrm{M}$ ) at $77 \mathrm{~K}$ (top), as well as polymer PDA and PNA at room temperature (RT) (bottom) excited at 280 and $360 \mathrm{~nm}$. c Polymer film excitation-phosphorescence mapping of PDA (top)

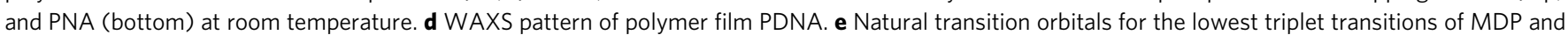
MND in gaseous state. $\mathbf{f}$ Proposed mechanism of the color-tunable UOP in multicomponent copolymer. Fluo. fluorescence, Phos. molecular phosphorescence, Exc. excitation.

interactions from the aggregated state can be detected. From the result of powder X-ray diffraction, only two small broad diffraction bands at around $17^{\circ}$ and $36^{\circ}$ were obtained, indicating the amorphous nature of PDNA (Supplementary Fig. 33). Furthermore, theoretical calculation of the polymer PDNA was conducted to verify the above speculation on color-tunable UOP (Fig. 3e), the lowest triplet state $\left(\mathrm{T}_{1}\right)$ of monomers MDP and MND is located at 362 and $498 \mathrm{~nm}$ in gaseous state, respectively, which is similar with the experimental data of 445 and $517 \mathrm{~nm}$.

Taken together, it was suggested that the color-tunable luminescence from blue to yellow was achieved in polymer PDNA by modulating the phosphorescence intensity ratio of blue emission of monomer MDP and yellow emission of monomer MND through variations in the excitation wavelengths (Fig. 3f). Meanwhile, numerous hydrogen bonds among the PAA chains form a rigid polymer microenvironment, which not only effectively restrict molecular motion to suppress the non-radiative decay of excited state but also prevent the quenching of triplet excitons by the surroundings (oxygen, moisture, etc.) for generating the ultralong lifetime phosphorescence emission. This mechanism can be further confirmed by an experiment regarding the effect of humidity on the lifetime of polymers (Supplementary Fig. 34 and Supplementary Table 5). When the polymer film PDNA was exposed to moist atmosphere (humidity is $86 \%$ ), the lifetime of PDNA at 445 and $517 \mathrm{~nm}$ exhibits a significant decrease within $60 \mathrm{~min}$, which may be attributed to the breakage of the hydrogen bonds among polymer chains by moisture. Furthermore, as a control, a multicomponent polymer was prepared by physically embedding the monomers MDP and MND into PAA with a molar ratio of 1:5:1000. The resulting polymer showed weak phosphorescence emission signals at 445 and $517 \mathrm{~nm}$ with a short lifetime, which further indicated that the covalent cross-linked network of the polymer played a critical role in color-tunable UOP (Supplementary Figs. 35 and 36).

General strategy for achieving color-tunable UOP. To establish the generality of our strategies for the generation of color-tunable UOP, another multicomponent copolymer PDBA was designed and prepared by radical cross-linked copolymerization of monomer di(but-3-en-1-yl) (1,1'-biphenyl)-4,4'-dicarboxylate (MBD), MDP, and AA with a molar feed ratio of 1:5:1000 (Fig. 4a and Supplementary Fig. 3). As expected, the polymer PDBA exhibited an excitation wavelength-dependent ultralong luminescence behavior under ambient conditions (Supplementary Movie 2). As shown in Fig. 4b, the emission bands of polymer PDBA displayed a gradual bathochromic shift from 445 to $514 \mathrm{~nm}$ when the excitation wavelength varied from 200 to $400 \mathrm{~nm}$, demonstrating color-tunable emission characteristics (Supplementary Figs. 37 and 38). In addition, the ultralong emission color spans from blue to green with a long lifetime of 578 and $609 \mathrm{~ms}$ at 445 and $514 \mathrm{~nm}$, respectively (Fig. 4c, e). Similar to PDNA, this color-tunable UOP property in the multicomponent copolymer PDBA is due to the dynamic ratio change of phosphorescence intensity from the different monomers MDP and MBD upon variations in the excitation wavelengths from 220 to $350 \mathrm{~nm}$ under ambient conditions (Fig. $4 \mathrm{f}$ and Supplementary Fig. 39). 

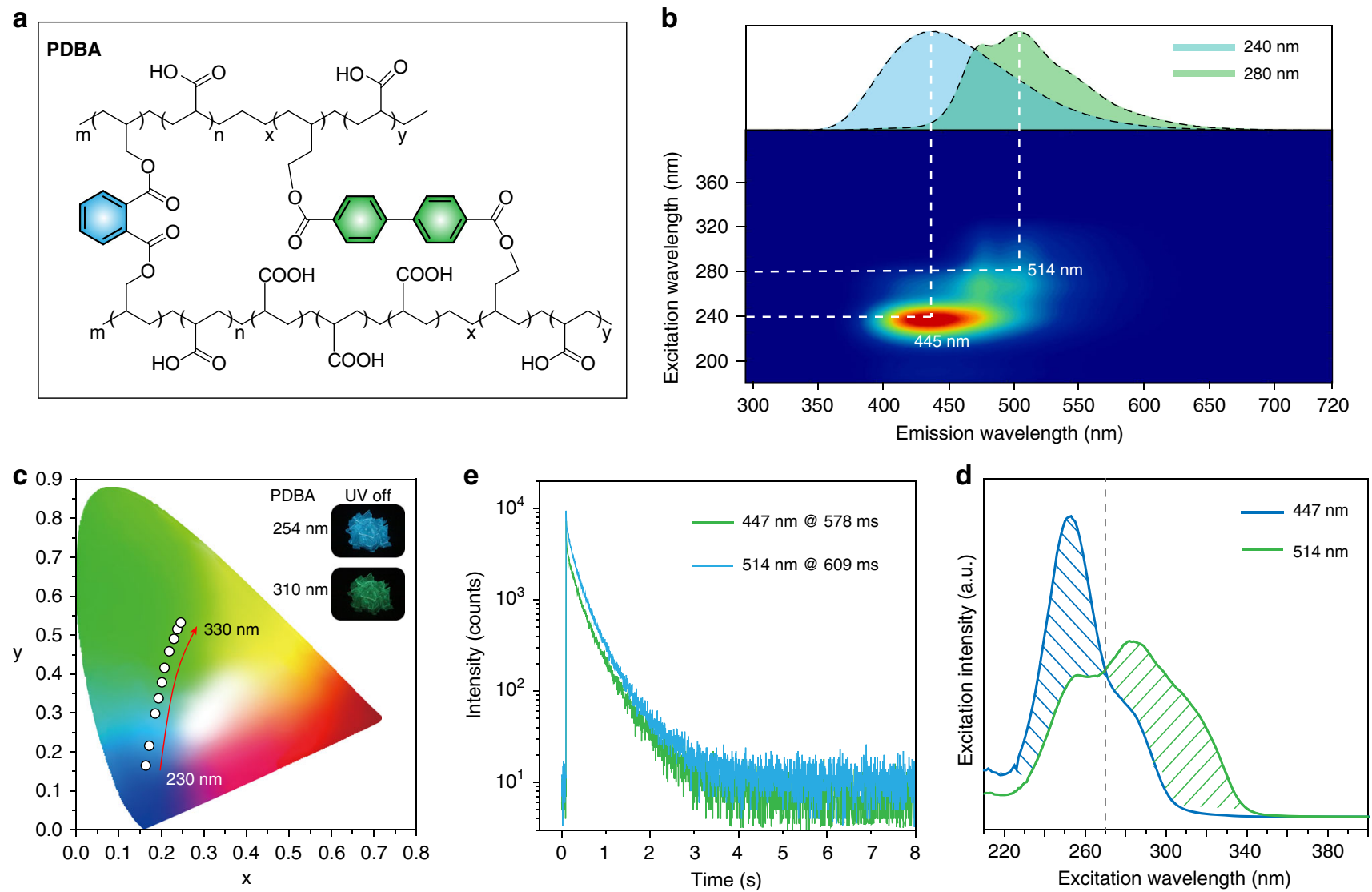

Fig. 4 Molecular structure and phosphorescence properties of the multicomponent copolymer PDBA under ambient conditions. a Molecular structure of polymer PDBA. $\mathbf{b}$ Excitation-phosphorescence mapping of PDBA film. The inset image shows the phosphorescence spectra of PDBA excited by corresponding excitation wavelengths. c CIE chromaticity diagram for polymer PDBA with excitation varied from $230 \mathrm{lo} 330 \mathrm{~nm}$. Inset: UOP photographs of polymer film PDBA excited at 254 and $310 \mathrm{~nm}$ after switching off the UV light, respectively. $\mathbf{d}$ Lifetime decay curves of bands at 447 and $514 \mathrm{~nm}$ in polymer PDBA, respectively. e Phosphorescence excitation spectra of polymer PDBA at 447 and $514 \mathrm{~nm}$.

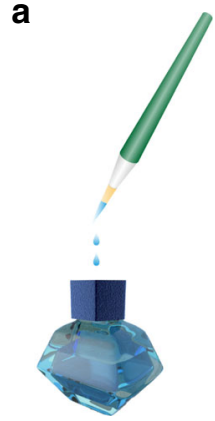

Encryption ink
Information encryption
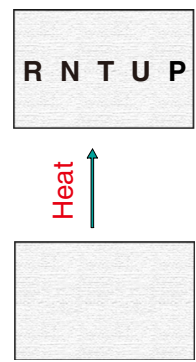

Information erasure
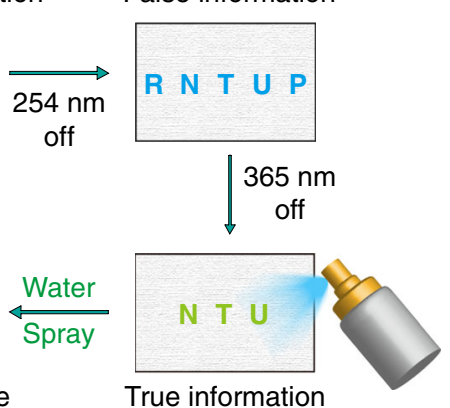

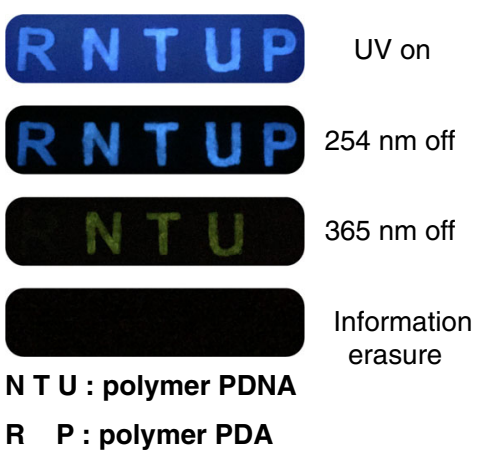

Fig. 5 Description of color-tunable UOP for multilevel information encryption. a Process of information encryption by using the multi-component copolymer PDNA (NTU) and PDA (RP) as encryption ink under ambient conditions. b Long-lived luminescence photographs of letters (RNTUP and NTU) before and after switching off the UV light of 254 and $365 \mathrm{~nm}$, respectively.

Potential application of color-tunable UOP. Given the excitationdependent UOP emission property in the polymer PDNA, a potential application in multilevel information encryption was explored. As demonstrated in Fig. 5, the encryption information pattern "RNTUP" was fabricated by using the polymers PDA and PDNA as encryption inks, and the pattern was left to dry for $10 \mathrm{~min}$ at $75^{\circ} \mathrm{C}$ in an oven. The letters "RP" and "NTU" show similar blue luminescence upon the UV irradiation at $254 \mathrm{~nm}$. After switching off the UV light, the pattern "RNTUP" with blue long-lived luminescence was observed by the naked eyes under ambient conditions.
The blue long-lived luminescence letters of "RNTUP" are false information. However, when the irradiation source changed from 254 to $365 \mathrm{~nm}$, the true information of "NTU" with yellow longlived phosphorescence emission can be captured after switching off the UV light. Therefore, using the excitation wavelength-responsive UOP nature of the polymer PDNA, multilevel information encryption can be achieved. Moreover, the information erasure function could be activated by spraying a small amount of water on the pattern. As shown in Fig. 5b, after spraying with water, the letters with phosphorescence luminescence signal disappeared when 
removed from the UV light of both 254 and $365 \mathrm{~nm}$, indicating that moisture can weaken the hydrogen-bonding network between polymer chains and enhance the molecular motion in polymer chains, which in turn quench room-temperature phosphorescence. This process can be easily reversed by drying the pattern in the oven, exhibiting the reusability of such materials. Visible and multilevel information encryption is essential for the development of secure information storage and anti-counterfeiting.

\section{Discussion}

In conclusion, we have presented an effective design strategy to achieve color-tunable UOP in single polymer through radical multicomponent cross-linked copolymerization. With the excitation wavelength changed from 254 to $370 \mathrm{~nm}$, the UOP emission color of copolymer turned from blue $(445 \mathrm{~nm})$ to yellow $(547 \mathrm{~nm})$ with an extensive emission zone in the visible spectrum under ambient conditions. Based on the results of various experiments and theoretical calculation, the dynamic ratiometric variation in phosphorescence intensity of different luminophores is responsible for the color-tunable UOP. Rigid cross-linked polymer network and hydrogen bonding among polymer chains effectively suppress the motion of polymer chains and prevent the quenching of triplet excitons by oxygen and moisture, creating conditions necessary for generating long-lived phosphorescence at room temperature. Notably, these multicomponent copolymers exhibit a lifetime of $1.2 \mathrm{~s}$ and the maximum phosphorescence quantum yield of $37.5 \%$ under ambient conditions. This study not only paves a fresh way to the design and preparation of colortunable UOP polymeric luminescence materials but also offers a guideline for developing materials toward multilevel information encryption, multicolor display, and biological applications.

\section{Methods}

Preparation of monomers and polymers. Diallyl phthalate (MDP): Phthalic acid $(0.50 \mathrm{~g} ; 3.01 \mathrm{mmol})$, 4-(dimethylamino)pyridine (DMAP, $0.88 \mathrm{~g} ; 7.22 \mathrm{mmol}$ ), $\mathrm{N}$-(3-dimethylaminopropyl)- $\mathrm{N}$-ethylcarbodiimide hydrochloride (EDCI) $(1.38 \mathrm{~g}$; $7.22 \mathrm{mmol})$, and prop-2-en-1-ol (0.38 g; $6.62 \mathrm{mmol})$ were dissolved in $\mathrm{N}, \mathrm{N}$-dimethylformamide $(100 \mathrm{~mL})$. After the solution was stirred at room temperature for $12 \mathrm{~h}$, the solvent was removed by rotary evaporation, and the residue was purified by column chromatography to give MDP $(0.42 \mathrm{~g}, 28.3 \%)$ as a faint yellow oil. ${ }^{1} \mathrm{H}$ NMR $\left(\mathrm{CDCl}_{3}\right): \delta$ 7.74-7.77 (m, 2H), 7.55-7.57 (m, 2H), 5.98-6.03 (m, 2H), $5.28-5.43(\mathrm{~m}, 4 \mathrm{H}), 4.80-4.83(\mathrm{~m}, 4 \mathrm{H}) .{ }^{13} \mathrm{C}$ NMR $\left(\mathrm{CDCl}_{3}\right): \delta 167.21,132.02,131.83$, $131.17,128.98,118.66,66.29$.

Di(but-3-en-1-yl) (1,1'-biphenyl)-4,4'-dicarboxylate (MBD): 1,1'-Biphenyl-4,4'dicarboxylic acid (0.50 g; $2.06 \mathrm{mmol})$, DMAP $(0.60 \mathrm{~g} ; 4.94 \mathrm{mmol})$, EDCI $(0.95 \mathrm{~g}$; $4.90 \mathrm{mmol})$, and but-3-en-1-ol $(0.33 \mathrm{~g} ; 4.54 \mathrm{mmol})$ were dissolved in $\mathrm{N}, \mathrm{N}$ dimethylformamide $(100 \mathrm{~mL})$. After the solution was stirred at room temperature for $12 \mathrm{~h}$, the solvent was removed by rotary evaporation, and the residue was purified by column chromatography to give MBD $(0.50 \mathrm{~g}, 34.5 \%)$ as a white solid. ${ }^{1} \mathrm{H} \mathrm{NMR}\left(\mathrm{CDCl}_{3}\right): \delta 8.11(\mathrm{~d}, 4 \mathrm{H}), 7.67(\mathrm{~d}, 4 \mathrm{H}), 5.93-5.83(\mathrm{~m}, 2 \mathrm{H}), 5.20-5.13(\mathrm{~m}$, $4 \mathrm{H}), 4.93(\mathrm{t}, 4 \mathrm{H}), 2.56-2.51(\mathrm{~m}, 4 \mathrm{H}){ }^{13} \mathrm{C} \mathrm{NMR}\left(\mathrm{CDCl}_{3}\right): \delta 166.32,144.44,134.10$, $130.28,129.96,127.32,117.57,64.19,33.28$.

Di(but-3-en-1-yl) naphthalene-2,6-dicarboxylate (MND): Naphthalene-2,6 dicarboxylic acid ( $0.50 \mathrm{~g} ; 2.31 \mathrm{mmol})$, DMAP $(0.67 \mathrm{~g} ; 5.54 \mathrm{mmol})$, EDCI (1.06 g; $5.54 \mathrm{mmol})$, and but-3-en-1-ol $(0.37 \mathrm{~g} ; 5.09 \mathrm{mmol})$ were dissolved in $\mathrm{N}, \mathrm{N}$ dimethylformamide $(100 \mathrm{~mL})$. After the solution was stirred at room temperature for $12 \mathrm{~h}$, the solvent was removed by rotary evaporation, and the residue was purified by column chromatography to give MND $(0.37 \mathrm{~g}, 25.9 \%)$ as a white solid. ${ }^{1} \mathrm{H}$ NMR $\left(\mathrm{CDCl}_{3}\right): \delta 8.67(\mathrm{~s}, 2 \mathrm{H}), 8.18(\mathrm{t}, 2 \mathrm{H}), 8.07(\mathrm{t}, 2 \mathrm{H}), 5.91-6.02(\mathrm{~m}, 2 \mathrm{H})$, $5.22-5.30(\mathrm{~m}, 4 \mathrm{H}), \delta 4.51(\mathrm{t}, 4 \mathrm{H}), 2.61-2.68(\mathrm{~m}, 4 \mathrm{H}) .{ }^{13} \mathrm{C} \mathrm{NMR}\left(\mathrm{CDCl}_{3}\right): \delta 166.39$, $134.66,134.04,130.68,129.76,129.66,126.08,117.59,64.45,33.29$.

PDA: The polymer was synthesized by radial copolymerization ${ }^{41}$. MDP $(0.02 \mathrm{~g}$, $0.081 \mathrm{mmol})$, acrylic acid $(5.86 \mathrm{~g}, 81.2 \mathrm{mmol})$, and AIBN $(0.006 \mathrm{~g})$ were dissolved in dry toluene $(75 \mathrm{~mL})$ under nitrogen atmosphere. After this solution was stirred at $70^{\circ} \mathrm{C}$ for $17 \mathrm{~h}$, the mixture was cooled to room temperature and the white solids were obtained by filtration. Then the crude product was washed with dichloromethane three times, which was dissolved in deionized water and dialyzed by dialysis tube (molecular weight cut-off $=2000$ ) for $72 \mathrm{~h}$. The solution was kept at $100^{\circ} \mathrm{C}$ for $6 \mathrm{~h}$, and the transparent polymer film was obtained.

PBA: Following the similar synthetic procedure for PDA, the reaction of MBD $(0.02 \mathrm{~g} ; 0.083 \mathrm{mmol})$, acrylic acid $(5.95 \mathrm{~g}, 82.57 \mathrm{mmol})$, and AIBN $(0.006 \mathrm{~g})$ in dry toluene solution $(75 \mathrm{~mL})$ for $17 \mathrm{~h}$ yielded PBA as a transparent film.
PNA: Following the similar synthetic procedure for PDA, the reaction of MND $(0.02 \mathrm{~g} ; 0.062 \mathrm{mmol})$, acrylic acid $(4.45 \mathrm{~g}, 61.67 \mathrm{mmol})$, and AIBN $(0.006 \mathrm{~g})$ in dry toluene solution $(75 \mathrm{~mL})$ for $17 \mathrm{~h}$ yielded PNA as a transparent film.

PDNA: Following the similar synthetic procedure for PDA, the reaction of MND (0.0013 g; $0.0041 \mathrm{mmol})$, MDP $(0.2 \mathrm{~g} ; 0.82 \mathrm{mmol})$, acrylic acid $(2.95 \mathrm{~g}$, $41.0 \mathrm{mmol})$, and AIBN $(0.008 \mathrm{~g})$ in dry toluene solution $(75 \mathrm{~mL})$ for $17 \mathrm{~h}$ yielded PDNA as a transparent film.

PDBA: Following the similar synthetic procedure for PDA, the reaction of MDP (0.045 g; $0.185 \mathrm{mmol})$, MBD $(0.013 \mathrm{~g} ; 0.037 \mathrm{mmol})$, acrylic acid $(2.60 \mathrm{~g}$, $37.0 \mathrm{mmol})$, and AIBN $(0.008 \mathrm{~g})$ in dry toluene solution $(75 \mathrm{~mL})$ for $17 \mathrm{~h}$ yielded PDBA as a transparent film.

\section{Data availability}

The authors declare that the data supporting the findings of this study are available within the article and its Supplementary Information. Extra data are available from the corresponding authors upon reasonable request.

Received: 26 September 2019; Accepted: 3 February 2020; Published online: 18 February 2020

\section{References}

1. Gu, L. et al. Colour-tunable ultra-long organic phosphorescence of a singlecomponent molecular crystal. Nat. Photon. 13, 406-411 (2019).

2. Maldiney, T. et al. The in vivo activation of persistent nanophosphors for optical imaging of vascularization, tumours and grafted cells. Nat. Mater. 13, 418-426 (2014).

3. Liu, X. et al. Binary temporal upconversion codes of $\mathrm{Mn}^{2+}$-activated nanoparticles for multilevel anti-counterfeiting. Nat. Commun. 8, 899 (2017).

4. Pan, Z., Lu, Y. \& Liu, F. Sunlight-activated long-persistent luminescence in the near-infrared from $\mathrm{Cr}^{3+}$-doped zinc gallogermanates. Nat. Mater. 11, 58-63 (2012)

5. Zhang, G., Palmer, G., Dewhirst, M. \& Fraser, C. A dual-emissive-materials design concept enables tumour hypoxia imaging. Nat. Mater. 8, 747-751 (2009).

6. Xu, S., Chen, R., Zheng, C. \& Huang, W. Excited state modulation for organic afterglow: materials and applications. Adv. Mater. 28, 9920-9940 (2016).

7. Gu, L. et al. Dynamic ultralong organic phosphorescence by photoactivation. Angew. Chem. Int. Ed. 57, 8425-8431 (2018).

8. Kwon, M. S., Lee, D., Seo, S., Jung, J. \& Kim, J. Tailoring intermolecular interactions for efficient room-temperature phosphorescence from purely organic materials in amorphous polymer matrices. Angew. Chem. Int. Ed. 53, 11177-11181 (2014).

9. Bian, L. et al. Simultaneously enhancing efficiency and lifetime of ultralong organic phosphorescence materials by molecular self-assembly. J. Am. Chem. Soc. 140, 10734-10739 (2018).

10. Kabe, R., Notsuka, N., Yoshida, K. \& Adachi, C. Afterglow organic lightemitting diode. Adv. Mater. 28, 655-660 (2016).

11. $\mathrm{Xu}, \mathrm{J} . \&$ Tanabe, S. Persistent luminescence instead of phosphorescence: history, mechanism, and perspective. J. Lumin. 205, 581-620 (2019).

12. Yuan, W. et al. Crystallization-induced phosphorescence of pure organic luminogens at room temperature. J. Phys. Chem. C 114, 6090-6099 (2010).

13. Shoji, Y. et al. Unveiling a new aspect of simple arylboronic esters: long-lived room-temperature phosphorescence from heavy-atom-free molecules. J. Am. Chem. Soc. 139, 2728-2733 (2017).

14. Xie, Y. et al. How the molecular packing affects the room temperature phosphorescence in pure organic compounds: ingenious molecular design, detailed crystal analysis, and rational theoretical calculations. Adv. Mater. 29, 1606829 (2017).

15. Cheng, Z. et al. Ultralong phosphorescence from organic ionic crystals under ambient conditions. Angew. Chem. Int. Ed. 57, 678-682 (2018).

16. Yang, Z. et al. Intermolecular electronic coupling of organic units for efficient persistent room-temperature phosphorescence. Angew. Chem. Int. Ed. 55, 2181-2185 (2016)

17. Hirata, S. et al. Efficient persistent room temperature phosphorescence in organic amorphous materials under ambient conditions. Adv. Funct. Mater. 23, 3386-3397 (2013)

18. Yang, X. \& Yan, D. Strongly enhanced long-lived persistent room temperature phosphorescence based on the formation of metal-organic hybrids. Adv. Optical Mater. 4, 897-905 (2016)

19. An, Z. et al. Stabilizing triplet excited states for ultralong organic phosphorescence. Nat. Mater. 14, 685-690 (2015).

20. Lucenti, E. et al. Cyclic triimidazole derivatives: intriguing examples of multiple emissions and ultralong phosphorescence at room temperature. Angew. Chem. Int. Ed. 56, 16302-16307 (2017). 
21. Bolton, O., Lee, K., Kim, H. J., Lin, K. Y. \& Kim, J. Activating efficient phosphorescence from purely organic materials by crystal design. Nat. Chem. 3, 205-210 (2011).

22. Zhang, Z., Chen, Y. \& Liu, Y. Efficient room-temperature phosphorescence of a solid-state supramolecule enhanced by cucurbit[6]uril. Angew. Chem. Int. Ed. 58, 6028-6032 (2019).

23. $\mathrm{Wu}, \mathrm{H}$. et al. Achieving amorphous ultralong room temperature phosphorescence by coassembling planar small organic molecules with polyvinyl alcohol. Adv. Funct. Mater. 29, 1807243 (2019).

24. Kenry, Chen,C. \& Liu, B. Enhancing the performance of pure organic room temperature phosphorescent luminophores. Nat. Commun. 10, 2111 (2019).

25. Gan, N., Shi, H., An, Z. \& Huang, W. Recent advances in polymer-based metal-free room-temperature phosphorescent materials. Adv. Funct. Mater. 28, 1802657 (2018).

26. Ma, X., Wang, J. \& Tian, H. Assembling-induced emission: an efficient approach for amorphous metal-free organic emitting materials with room temperature phosphorescence. Acc. Chem. Res. 52, 738-748 (2019).

27. Ogoshi, T. et al. Ultralong room-temperature phosphorescence from amorphous polymer poly(styrene sulfonic acid) in air in the dry solid state. Adv. Funct. Mater. 28, 1707369 (2018)

28. Ma, X., Xu, C., Wang, J. \& Tian, H. Amorphous pure organic polymers for heavy-atom-free efficient room-temperature phosphorescence emission. Angew. Chem. Int. Ed. 57, 10854-10858 (2018).

29. Su, Y. et al. Ultralong room temperature phosphorescence from amorphous organic materials toward confidential information encryption and decryption. Sci. Adv. 4, eaas9732 (2018).

30. Deng, R. et al. Temporal full-colour tuning through non-steady-state upconversion. Nat. Nanotechnol. 10, 237-242 (2015).

31. Lee, H., Kim, J., Kim, H., Kim, J. \& Kwon, S. Colour-barcoded magnetic microparticles for multiplexed bioassays. Nat. Mater. 9, 745-749 (2010).

32. Lee, J. et al. Universal process-inert encoding architecture for polymer microparticles. Nat. Mater. 13, 524-529 (2014).

33. Pan, L. et al. Truly fluorescent excitation-dependent carbon dots and their applications in multicolor cellular imaging and multidimensional sensing. Adv. Mater. 27, 7782-7787 (2015).

34. He, G. et al. A Color-tunable europium complex emitting three primary colors and white light. Angew. Chem. Int. Ed. 48, 6132-6135 (2009).

35. Li, W. et al. A universal strategy for activating multi-color room temperature afterglow of carbon dots in boric acid matrix. Angew. Chem. Int. Ed. 58, 7278-7283 (2019).

36. Zhang, Y. et al. Excitation wavelength-dependent fluorescence of an ESIPT triazole derivative for amine sensing and anti-counterfeiting applications. Angew. Chem. Int. Ed. 58, 8773-8778 (2019).

37. Wang, J. et al. Multicolor emission from non-conjugated polymers based on a single switchable boron chromophore. Angew. Chem. Int. Ed. 58, 3082-3086 (2019).

38. Lee, K. et al. Excitation-dependent visible fluorescence in decameric nanoparticles with monoacylglycerol cluster chromophores. Nat. Commun. 4, 1544 (2013).

39. $\mathrm{Hu}$, J. et al. Developing through-space charge transfer polymers as a general approach to realize full-color and white emission with thermally activated delayed fluorescence. Angew. Chem. Int. Ed. 58, 8405-8409 (2019).

40. Mohamed, R. R., Abu Elella, M. H. \& Sabaa, M. W. Cytotoxicity and metal ions removal using antibacterial biodegradable hydrogels based on
$\mathrm{N}$-quaternized chitosan/poly(acrylic acid). Int. J. Biol. Macromol. 98, 302-313 (2017)

41. Zhou, Q. et al. Emission mechanism understanding and tunable persistent room temperature phosphorescence of amorphous nonaromatic polymers. Mater. Chem. Front. 3, 257-264 (2019).

\section{Acknowledgements}

The research is supported by the Singapore Agency for Science, Technology and Research (A*STAR) AME IRG grant (A1883c0005), the Singapore National Research Foundation Investigatorship (NRF-NRFI2018-03), and partially by the National Key Research and Development Program of China (2017YFA0207700).

\section{Author contributions}

L.G., H.W.W., W.P.Y., Z.F.A., and W.H. conceived the experiments. L.G. and Y.L.Z. wrote the manuscript. W.Y.J. measured the quantum efficiency. H.L.M. contributed to theoretical calculations. H.Z.C., N.Z., C.Q., H.W., and D.D.W. were primarily responsible for the experiments. All authors contributed to the data analyses.

\section{Competing interests}

The authors declare no competing interests.

\section{Additional information}

Supplementary information is available for this paper at https://doi.org/10.1038/s41467 020-14792-1.

Correspondence and requests for materials should be addressed to Z.F.A., W.H. or Y.L.Z.

Peer review information Nature Communications thanks He Tian, Guoqing Zhang, and the other anonymous reviewer(s) for their contribution to the peer review of this work.

Reprints and permission information is available at http://www.nature.com/reprints

Publisher's note Springer Nature remains neutral with regard to jurisdictional claims in published maps and institutional affiliations.

\begin{abstract}
cC (i) Open Access This article is licensed under a Creative Commons Attribution 4.0 International License, which permits use, sharing, adaptation, distribution and reproduction in any medium or format, as long as you give appropriate credit to the original author(s) and the source, provide a link to the Creative Commons license, and indicate if changes were made. The images or other third party material in this article are included in the article's Creative Commons license, unless indicated otherwise in a credit line to the material. If material is not included in the article's Creative Commons license and your intended use is not permitted by statutory regulation or exceeds the permitted use, you will need to obtain permission directly from the copyright holder. To view a copy of this license, visit http://creativecommons.org/ licenses/by/4.0/.
\end{abstract}

(C) The Author(s) 2020 